\title{
Solution of Linear Fuzzy Fractional Differential Equations Using Fuzzy Natural Transform
}

\author{
Hameeda Oda Al-Humedi ${ }^{1, *}$ and Shaimaa Abdul-Hussein Kadhim ${ }^{2}$ \\ 1,2 Department of Mathematics, College of Education for Pure Sciences, University of Basrah, \\ Basrah, Iraq \\ e-mail: hameeda.mezban@uobasrah.edu.iq ${ }^{1}$; shaimaa.a_hussein@yahoo.com²
}

\begin{abstract}
The purpose of this paper is to apply the fuzzy natural transform (FNT) for solving linear fuzzy fractional ordinary differential equations (FFODEs) involving fuzzy Caputo's Hdifference with Mittag-Leffler laws. It is followed by proposing new results on the property of FNT for fuzzy Caputo's H-difference. An algorithm was then applied to find the solutions of linear FFODEs as fuzzy real functions. More specifically, we first obtained four forms of solutions when the FFODEs is of order $\alpha \in(0,1]$, then eight systems of solutions when the FFODEs is of order $\alpha \in(1,2]$ and finally, all of these solutions are plotted using MATLAB. In fact, the proposed approach is an effective and practical to solve a wide range of fractional models.
\end{abstract}

\section{Introduction}

The subject of fractional calculus, a generality of the ordinary calculus, described when the order of the functions' derivative is non-integer. Fractional calculus has a long history, where, explored and studied by various researchers in different fields of mathematics, engineering and physics have been intensively described via fractional derivatives, and fractional differential equations have come up as powerful instrument for modeling many difficult types of complex problems. Many authors considered the terms such as Riemann-Liouville, Grüunwald-Letnikov and Caputo fractional derivative when dealing with fractional differential equations ([3], [23], [29], [40]). As times moving on, some drawbacks seem in the fractional differential equations especially, when dealing

Received: September 29, 2021; Accepted: November 1, 2021

2020 Mathematics Subject Classification: 30C55, 30C45.

Keywords and phrases: Caputo's H-difference, fuzzy fractional differential equations, fuzzy natural transform, Mittag-Leffler function. 
with physical phenomena. One of which is that a model involves uncertainty quantities and thus it is difficult to determine the initial values of it, as seen in ([13], [39]). To handle uncertainty quantities, researchers suggest several concepts. What stands out among those concepts is fuzzy set theory [44]. This theory is able to deal with differential equations having uncertainties at initial values. The first contribution on handling fractional differential equations with uncertainty was studied in [3]. This has prompted many researchers to further explore the topic ([4]-[6], [34], [23], [41]). It is also possible to solve many types of equations using integral transforms. Where integral transforms were used in solving linear ordinary differential equations, as well as linear fractional differential equations. The integral transforms were preceded by Fourier transform. Following, several integral transforms have been proposed, namely, Laplace, Mellin, Hankel and Sumudu transforms ([17], [24], [27], [32]). In this paper, we go to apply the natural transform to solve linear fuzzy fractional ordinary differential equations FFODEs involving fuzzy Caputo's H-difference. The natural transform is a new integral transform was introduced by [10], and its properties were giving by [38] also they proved that the natural transform method converges to the Laplace and Sumudu transforms. This transform was applied to solve the ordinary differential equations ([20], [28]). Moreover, in [28], some certain fundamental properties of generalized natural transform in generalized spaces were established. In [37], this new transform was applied to find analytical solutions of fractional-order heat and wave equations. In the recent years and with the rapid development of linear and nonlinear science, many effectiveness numerical and analytical different methods for solving the fuzzy fractional differential equations, as using the fuzzy Laplace and fuzzy Sumudu transforms, see ([1], [14]). The objective of this paper is to discuss the numerically solutions for each form and system of FFODEs based on orders of fuzzy Caputo's H-difference by using FNT.

The paper is presented as follows: Section 2 gives the fundamental definitions and theorems of fuzzy calculus, fuzzy fractional derivatives and the types of Mittag-Leffler functions. The definition of fuzzy natural transform FNT for Caputo's H-difference is given in Section 3. Algorithm of the solution of linear fuzzy fractional ordinary differential equations FFODEs of order $0<\alpha \leq 1$ and $1<\alpha \leq 2$ is presented in Section 4. The solutions of the FFODEs by four forms and eight systems are described in Section 5 , respectively. Section 6 focuses on the results of the numerical applications are performed to test the validity and reliability of the present method in Section 4. Finally, the conclusions are drawn in Section 7. 


\section{Basics Concepts}

Fuzzy Calculus 2.1. In this subsection, several essential definitions and theorems of fuzzy calculus will be introduced.

Definition 2.2. A fuzzy number $u$ is a pair $(\underline{u}, \bar{u})$ of functions $\underline{u}(r), \bar{u}(r), 0 \leq r \leq 1$ which satisfy the following requests [36]:

i: $\underline{u}(r)$ is bounded monotonic increasing left continuous function.

ii: $\bar{u}(r)$ is bounded monotonic decreasing left continuous function.

iii: $\underline{u}(r) \leq \bar{u}(r), \quad 0 \leq r \leq 1$

Arithmetic operation of arbitrary fuzzy numbers $u=(\underline{u}(r), \bar{u}(r))$ and $v=$ $(\underline{v}(r), \bar{v}(r)) \in \mathbb{R}$, can be defined as [1],

(a) $u=v$, if and only if $\underline{u}(r)=\underline{v}(r)$ and $\bar{u}(r)=\bar{v}(r)$.

(b) $u+v=(\underline{u}(r)+\underline{v}(r), \bar{u}(r)+\bar{v}(r))$.

(c) $u-v=(\underline{u}(r)-\bar{v}(r), \bar{u}(r)-\underline{v}(r))$.

(d) $\lambda u= \begin{cases}(\lambda \underline{u}(r), \lambda \bar{u}(r)), & \lambda \geq 0, \\ (\lambda \underline{u}(r), \lambda \bar{u}(r)), & \lambda<0 .\end{cases}$

The fuzzy numbers are classified into several types of fuzzy membership function. The definition of triangular fuzzy number will be mentioned, because it's the most used membership functions in our study.

Definition 2.3 [14]. In a triangular fuzzy number (TFN) is defined by notation $A=[a, b, c]$. Then the members help function for this TFN is defined as

$$
A(t)=\left\{\begin{array}{cc}
0, & t<a \\
\frac{t-a}{b-a}, & a \leq t \leq b \\
\frac{c-t}{c-b}, & b \leq t \leq c
\end{array} .\right.
$$

The $r$-cuts of TFN is $A^{r}=[(b-a) r+a(b-c) r+c], \forall r \in(0,1]$. 
Theorem 2.4 [15]. Let $F(t): \mathbb{R} \rightarrow \mathcal{F}(\mathbb{R})$ and it is denoted by $\left[\underline{f}_{r}(t), \bar{f}_{r}(t)\right]$. So for any fixed $r \in[0,1]$, imagine $\underline{f}_{r}(t)$ and $\bar{f}_{r}(t)$ are Riemann-integrable on $[a, b]$ for every $b \geq a$, and two positive $\underline{M}_{r}$ and $\bar{M}_{r}$ such that, $\int_{a}^{b}\left|\underline{f}_{r}(t)\right| d x \leq \underline{M}_{r}$ and $\int_{a}^{b}\left|\bar{f}_{r}(t)\right| d x \leq$ $\bar{M}_{r}$ for every $b \geq a$. Then, $F(t)$ is inadvisable fuzzy Riemann-integrable on $[a, \infty)$ and it is a fuzzy number. Thereby,

$$
\int_{a}^{\infty} F(t) d x=\left[\int_{a}^{\infty} \underline{f}_{r}(t) d x, \int_{a}^{\infty} \bar{f}_{r}(t) d x\right] .
$$

Hukuhara (H-difference) of fuzzy number is defined as follows.

Definition 2.5 [36]. If $u, v \in \mathcal{F}(\mathbb{R})$ and exists a fuzzy subset $w \in \mathcal{F}(\mathbb{R})$ such as $w+u=v$, thereby $w$ is unique. In this case, $w$ is called H-difference, of $u$ and $v$ denoted by $v-{ }_{H} u$ and also note that $-_{H} u \neq u+(-1) v$.

The concept of strongly generalized differentiable is a popularization of $\mathrm{H}$-difference for fuzzy functions, it is defined as follows.

Definition 2.6 [43]. Let $F(t):(a, b) \rightarrow \mathcal{F}(\mathbb{R})$ be a fuzzy function and $t_{0} \in(a, b)$. That $F(t)$ is strongly generalized differentiable at $t_{0}$ if there exists an element $F^{\prime}\left(t_{0}\right) \in$ $\mathcal{F}(\mathbb{R})$, then:

For all $h>0$ sufficiently small, $\exists F\left(t_{0}+h\right)-{ }_{H} F\left(t_{0}\right), \exists F\left(t_{0}\right)-{ }_{H} F\left(t_{0}-h\right)$, and the limits (in the metric $D$ )

$$
\lim _{h \rightarrow 0} \frac{F\left(t_{0}+h\right)-{ }_{H} F\left(t_{0}\right)}{h}=\lim _{h \rightarrow 0} \frac{F\left(t_{0}\right)-{ }_{H} F\left(t_{0}-h\right)}{h}=F^{\prime}\left(t_{0}\right) .
$$

Or,

For every $h>0$ enough small, $\exists F\left(t_{0}\right)-{ }_{H} F\left(t_{0}+h\right), \exists F\left(t_{0}-h\right)-{ }_{H} F\left(t_{0}\right)$ and so the limits (in the metric $D$ )

$$
\lim _{h \rightarrow 0} \frac{F\left(t_{0}\right)-{ }_{H} F\left(t_{0}+h\right)}{-h}=\lim _{h \rightarrow 0} \frac{F\left(t_{0}-h\right)-{ }_{H} F\left(t_{0}\right)}{-h}=F^{\prime}\left(t_{0}\right) .
$$

The symbols $\mathbb{C}_{\mathcal{F}}[a, b]$ and $L_{\mathcal{F}}[a, b]$ are used to refer for the spaces of all continuous fuzzy functions and Lebesgue integrable fuzzy functions, respectively on $[a, b] \subseteq \mathbb{R}$.

Fuzzy Fractional Derivatives 2.7. In this subsection, some of these definitions and theorems are presented on the subject of fuzzy Caputo's H-difference. This subject extended of crisp case for fuzzy discussed in [35]. 
Lemma 2.8. Let $f(t)$ be a crisp continuous function and $(\lceil\alpha\rceil)$-time differentiable in the independent variable $t$ over the interval of differentiation (integration) $[0, t]$. Then the relation,

$$
{ }^{C} D^{\alpha} f(t)={ }^{R L} D^{\alpha}\left(f(t)-\sum_{p=0}^{\lfloor\alpha\rfloor} \frac{t^{p}}{p !} f_{0}^{(p)}\right), \quad \alpha \in(n-1, n], \quad n \in \mathbb{N},
$$

holds, where $f_{0}^{(p)}=\left.\frac{d^{p} f(t)}{d t^{p}}\right|_{t=0}$ and ${ }^{c} D^{\alpha}$ means Caputo derivative operator, while $\lceil\alpha\rceil$ and $\lfloor\alpha\rfloor$ are the value $\alpha$ rounded up and down to the closet integer number, respectively. ${ }^{R L} D^{\alpha}$ is the common Riemann-Liouville fractional derivative operator which is defined as follows:

$$
{ }^{R L} D^{\alpha} f(t)=\frac{1}{\Gamma(\lceil\alpha\rceil-\alpha)} \frac{d^{\lceil\alpha\rceil}}{d t^{\lceil\alpha\rceil}} \int_{0}^{t} \frac{f(s)}{(t-s)^{1-[\alpha]+\alpha}} d s
$$

Definition 2.9. Let $F(t) \in \mathbb{C}_{\mathcal{F}}[0, b] \cap L_{\mathcal{F}}[0, b], K(t)=\frac{1}{\Gamma([\alpha]-\alpha)} \int_{0}^{t} \frac{t^{f(s)-\sum_{p=0}^{\mid \alpha]} \frac{s_{p}^{p}}{p !} f_{0}^{(p)}}}{(t-s)^{1-[\alpha]+\alpha}} d s$, and

$$
\lim _{h \rightarrow 0^{+}} \frac{K\left(t_{0}+h\right)-{ }_{H} K\left(t_{0}\right)}{h}=\lim _{h \rightarrow 0^{+}} \frac{K\left(t_{0}\right)-{ }_{H} K\left(t_{0}-h\right)}{h}=Q\left(t_{0}\right),
$$

and

$$
\lim _{h \rightarrow 0^{+}} \frac{K\left(t_{0}\right)-{ }_{H} K\left(t_{0}+h\right)}{-h}=\lim _{h \rightarrow 0^{+}} \frac{K\left(t_{0}-h\right)-{ }_{H} K\left(t_{0}\right)}{-h}=L\left(t_{0}\right)
$$

$F(t)$ is Caputo's $\mathrm{H}$-difference function of order $0<\alpha<2, \alpha \neq 1$ at $t_{0} \in(0, b)$, if there exists an element ${ }^{C} D^{\alpha} F\left(t_{0}\right) \in \mathbb{C}_{\mathcal{F}}$ such that for every $0 \leq r \leq 1$ and for $h>0$ adequately near zero, either,

(a) $\lim _{h \rightarrow 0^{+}} \frac{K\left(t_{0}+h\right)-{ }_{H} K\left(t_{0}\right)}{h}=\lim _{h \rightarrow 0^{+}} \frac{K\left(t_{0}\right){ }_{H} K\left(t_{0}-h\right)}{h}={ }^{C} D^{\alpha} F\left(t_{0}\right)$.

(b) $\lim _{h \rightarrow 0^{+}} \frac{K\left(t_{0}\right){ }_{H} K\left(t_{0}+h\right)}{-h}=\lim _{h \rightarrow 0^{+}} \frac{K\left(t_{0}-h\right)-{ }_{H} K\left(t_{0}\right)}{-h}={ }^{C} D^{\alpha} F\left(t_{0}\right)$, for $0<\alpha<1$.

(c) $\lim _{h \rightarrow 0^{+}} \frac{Q\left(t_{0}+h\right)-{ }_{H} Q\left(t_{0}\right)}{h}=\lim _{h \rightarrow 0^{+}} \frac{Q\left(t_{0}\right)-{ }_{H} Q\left(t_{0}-h\right)}{h}={ }^{C} D^{\alpha} F\left(t_{0}\right)$.

(d) $\lim _{h \rightarrow 0^{+}} \frac{Q\left(t_{0}\right)-{ }_{H} Q\left(t_{0}+h\right)}{-h}=\lim _{h \rightarrow 0^{+}} \frac{Q\left(t_{0}-h\right){ }_{H} Q\left(t_{0}\right)}{-h}={ }^{C} D^{\alpha} F\left(t_{0}\right)$. 
(e) $\lim _{h \rightarrow 0^{+}} \frac{L\left(t_{0}+h\right)-{ }_{H} L\left(t_{0}\right)}{h}=\lim _{h \rightarrow 0^{+}} \frac{L\left(t_{0}\right)-{ }_{H} L\left(t_{0}-h\right)}{h}={ }^{C} D^{\alpha} F\left(t_{0}\right)$.

(f) $\lim _{h \rightarrow 0^{+}} \frac{L\left(t_{0}\right)-{ }_{H} L\left(t_{0}+h\right)}{-h}=\lim _{h \rightarrow 0^{+}} \frac{L\left(t_{0}-h\right)-{ }_{H} L\left(t_{0}\right)}{-h}={ }^{C} D^{\alpha} F\left(t_{0}\right)$, for $1<\alpha<2$.

Remark 2.10. The fuzzy function $F(t)$ is Caputo's H-difference and denoted by ${ }^{c} D_{1}^{\alpha} F(t),{ }^{c} D_{2}^{\alpha} F(t)$ respectively if it is $(n)$-differentiable for $n=1,2$ as in Definition 2.9 cases $(\mathbf{a}, \mathbf{b})$. Also, the fuzzy function $F(t)$ is Caputo's H-difference and denoted by ${ }^{c} D_{1,1}^{\alpha} F(t),{ }^{c} D_{1,2}^{\alpha} F(t),{ }^{c} D_{2,1}^{\alpha} F(t),{ }^{c} D_{2,2}^{\alpha} F(t)$ respectively if it is $(n, m)-$ differentiable for $n, m=1,2$ as in Definition 2.9 cases(c,d,e,f).

Theorem 2.11. Let $F(t) \in \mathbb{C}_{\mathcal{F}}[0, b] \cap L_{\mathcal{F}}[0, b]$ be a fuzzy valued function and where $[F(t)]^{r}=\left[\underline{f}_{r}(t), \bar{f}_{r}(t)\right]$, for $r \in[0,1]$ and $t_{0} \in(0, b)$. Then:

i- If $F(t)$ is Caputo's H-difference function in (1)-differentiable form, then for $0<\alpha<1$

$$
\left[{ }^{c} D_{1}^{\alpha} F\left(t_{0}\right)\right]^{r}=\left[{ }^{c} D^{\alpha} \underline{f}_{r}\left(t_{0}\right),{ }^{c} D^{\alpha} \bar{f}_{r}\left(t_{0}\right)\right] .
$$

ii- If $F(t)$ is Caputo's H-difference function in (2)-differentiable form, then for $0<\alpha<1$

$$
\left[{ }^{c} D_{2}^{\alpha} F\left(t_{0}\right)\right]^{r}=\left[{ }^{c} D^{\alpha} \overline{f_{r}}\left(t_{0}\right),{ }^{c} D^{\alpha} \underline{f_{r}}\left(t_{0}\right)\right] .
$$

iii- If ${ }^{c} D_{1}^{\alpha} F(t)$ is Caputo's H-difference function in $(1,1)$-differentiable form, then for $1<\alpha<2$

$$
\left[{ }^{c} D_{1,1}^{\alpha} F\left(t_{0}\right)\right]^{r}=\left[{ }^{c} D^{\alpha} \underline{f}_{r}\left(t_{0}\right),{ }^{c} D^{\alpha} \bar{f}_{r}\left(t_{0}\right)\right] .
$$

iv- If ${ }^{c} D_{1}^{\alpha} F(t)$ is Caputo's H-difference function in (1,2)-differentiable form, then for $1<\alpha<2$

$$
\left[{ }^{c} D_{1,2}^{\alpha} F\left(t_{0}\right)\right]^{r}=\left[{ }^{c} D^{\alpha} \overline{f_{r}}\left(t_{0}\right),{ }^{c} D^{\alpha} \underline{f_{r}}\left(t_{0}\right)\right] .
$$

$\mathbf{v}$ - If ${ }^{c} D_{2}^{\alpha} F(t)$ is Caputo's H-difference function in (2,1)-differentiable form, then for $1<\alpha<2$

$$
\left[{ }^{c} D_{2,1}^{\alpha} F\left(t_{0}\right)\right]^{r}=\left[{ }^{c} D^{\alpha} \overline{f_{r}}\left(t_{0}\right),{ }^{c} D^{\alpha} \underline{f_{r}}\left(t_{0}\right)\right] .
$$


vi- If ${ }^{c} D_{2}^{\alpha} F(t)$ is Caputo's H-difference function in (2,2)-differentiable form, then for $1<\alpha<2$

$$
\left[{ }^{c} D_{2,2}^{\alpha} F\left(t_{0}\right)\right]^{r}=\left[{ }^{c} D^{\alpha} \underline{f}_{r}\left(t_{0}\right),{ }^{c} D^{\alpha} \bar{f}_{r}\left(t_{0}\right)\right] .
$$

where

$$
\begin{gathered}
{ }^{c} D^{\alpha} \underline{f_{r}}\left(t_{0}\right)=\left[\frac{1}{\Gamma([\alpha]-\alpha)} \int_{0}^{t} \frac{D^{[\alpha]} \tilde{r}_{r}(t)}{(t-s)^{1-[\alpha]+\alpha}} d s\right]_{t=t_{0}}, \\
{ }^{c} D^{\alpha} \overline{f_{r}}\left(t_{0}\right)=\left[\frac{1}{\Gamma([\alpha]-\alpha)} \int_{0}^{t} \frac{D^{[\alpha]} \overline{r_{r}}(t)}{(t-s)^{1-[\alpha]+\alpha}} d s\right]_{t=t_{0}} . \\
D^{k} f(t)=\frac{d^{k} f(t)}{d t^{k}} .
\end{gathered}
$$

Mittag-Leffler Function 2.12. This function is a direct generalization of the exponential function, and has an affinity for fractional calculus. In this subsection, one and two parameter representations of Mittag-Leffler function are presented by the following definitions [22].

Definition 2.13. A one-parameter function of Mittag-Leffler type defined by the series expansion

$$
E_{\alpha}(z)=\sum_{k=0}^{\infty} \frac{z^{k}}{\Gamma(\alpha k+1)}, \quad \alpha>0, \quad z \in \mathbb{C} .
$$

Definition 2.14. A two-parameter function of Mittag-Leffler type defined by the series expansion

$$
E_{\alpha, \beta}(z)=\sum_{k=0}^{\infty} \frac{z^{k}}{\Gamma(\alpha k+\beta)}, \quad \alpha>0, \beta>0, \quad z \in \mathbb{C} .
$$

Consequently, the definition for the classical natural transform is given, when dealing with Caputo's fractional derivative of crisp type and then some properties.

Theorem 2.15 [26]. Let $n \in \mathbb{N}$ and $\alpha>0$ be such that $n-1<\alpha \leq n$ and $R^{+}(s, u)$ be a natural transform of the function $f(t)$. Then the natural transform denoted by $R_{\alpha}^{+}(s, u)$ of Caputo fractional derivative of the function $f(t)$ of order $\alpha$, is given by

$$
N^{+}\left[{ }^{c} D^{\alpha} f(t)\right]=R_{\alpha}^{+}(s, u)=\frac{s^{\alpha}}{u^{\alpha}} R^{+}(s, u)-\sum_{k=0}^{n-1} \frac{s^{\alpha-(k+1)}}{u^{\alpha-k}}\left[D^{k} f(t)\right]_{t=0} .
$$


Since in this paper, the orders $0<\alpha \leq 1$ and $1<\alpha \leq 2$ are considered, Theorem 2.16 can be simplified as

$$
\begin{aligned}
& N^{+}\left[{ }^{c} D^{\alpha} f(t)\right]=R_{\alpha}^{+}(s, u)=\frac{s^{\alpha}}{u^{\alpha}} R^{+}(s, u)-\frac{s^{\alpha-1}}{u^{\alpha}} D^{0} f(0), \alpha \in(0,1], \\
& N^{+}\left[{ }^{c} D^{\alpha} f(t)\right]=R_{\alpha}^{+}(s, u)=\frac{s^{\alpha}}{u^{\alpha}} R^{+}(s, u)-\frac{s^{\alpha-1}}{u^{\alpha}} D^{0} f(0)-\frac{s^{\alpha-2}}{u^{\alpha-1}} D^{1} f(0), \alpha \in(1,2] .
\end{aligned}
$$

Note that when $\alpha=1$ and $\alpha=2$, the definition is like to the definition of natural transform for first and second orders derivative.

Property 2.16 [26]. If $\alpha>-1$, then the natural transform of $t^{\alpha}$ is given by

$$
N^{+}\left[t^{\alpha}\right]=\frac{\Gamma(\alpha+1) u^{\alpha}}{s^{\alpha+1}} .
$$

Property 2.17 [18]. If $\alpha, \beta>0, \alpha \in \mathbb{R}$ and $\frac{s^{\alpha}}{u^{\alpha}}>|a|$, then the inverse natural transform formula,

$$
N^{-1}\left[\frac{u^{\beta-1} s^{\alpha-\beta}}{s^{\alpha}+a u^{\alpha}}\right]=t^{\beta-1} E_{\alpha, \beta}\left(-a t^{\alpha}\right) .
$$

\section{Fuzzy Natural transforms for Caputo's H-difference}

The definition of FNT for Caputo's H-difference will be introduced as follows ([26], [33]).

Definition 3.1. Let $F(t): \mathbb{R} \rightarrow \mathcal{F}(\mathbb{R})$ be a continuous fuzzy function. Assume $F(u t) e^{-s t}$ is improper fuzzy Riemann-integrable on $[0, \infty)$, then $\int_{0}^{\infty} F(u t) e^{-s t} d t$ is called fuzzy Natural transform and is denoted by,

$$
R(s, u)=N^{+}[F(t)](u, s)=\frac{1}{u} \int_{0}^{\infty} F(u t) e^{-s t} d t, \quad s, u \in\left[-\vartheta_{1}, \vartheta_{2}\right]
$$

where, the variables $u$ and $s$ are used to factor the variable $t$ in the argument of the fuzzy and $\vartheta_{1}, \vartheta_{2}>0$. Also, the FNT can be written in the following form:

$$
N^{+}[F(t)](u, s)=\left[N^{+}\left[\underline{f}_{r}\left(t_{0}\right)\right](u, s), \underline{f}_{r}\left(t_{0}\right), N^{+}\left[\bar{f}_{r}\left(t_{0}\right)\right](u, s)\right] .
$$

\section{Algorithm of the Solution of Linear Fuzzy Fractional Ordinary Differential Equations (FFODEs)}

The FFODEs is given in this form: 


$$
\left[{ }^{C} D^{\alpha} \tilde{y}(t)\right]^{r}=a[\tilde{y}(t)]^{r}+b,
$$

where, ${ }^{C} D^{\alpha}$ is Caputo's H-difference of order, $0<\alpha \leq 1$ or $1<\alpha \leq 2, r \in[0,1]$ and $a, b$ are constants. The fuzzy initial condition FIC is the triangular fuzzy number:

$$
[\tilde{y}(0)]^{r}=\left[S_{1}, S_{2}, S_{3}\right]
$$

where, $[\tilde{y}(t)]^{r}$ is a fuzzy function of the crisp function $y(t)$. Then Eq. (1) and Eq. (2) are equivalent the following forms, respectively:

$$
\begin{gathered}
{\left[{ }^{c} D^{\alpha} \underline{y}_{r}(t),{ }^{c} D^{\alpha} \bar{y}_{r}(t)\right]=a\left[\underline{y}_{r}(t), \bar{y}_{r}(t)\right]+b,} \\
{\left[\underline{y}_{r}(0), \bar{y}_{r}(0)\right]=\left[S_{1}+r\left(S_{2}-S_{1}\right), S_{3}-r\left(S_{3}-S_{2}\right)\right] .}
\end{gathered}
$$

Next, the algorithm for solving of FFODEs can be provided through the following steps:

Step 1: Eq. (3) is written in the form of a system consisting of two equations. The same procedure which applied to Eq.(4) as follows:

$$
\begin{aligned}
& { }^{c} D^{\alpha} \underline{y}_{r}(t)=a \underline{y}_{r}(t)+b, \\
& { }^{c} D^{\alpha} \bar{y}_{r}(t)=a \bar{y}_{r}(t)+b,
\end{aligned}
$$

and

$$
\begin{aligned}
& \underline{y_{r}}(0)=S_{1}+r\left(S_{2}-S_{1}\right), \\
& \bar{y}_{r}(0)=S_{3}-r\left(S_{3}-S_{2}\right) .
\end{aligned}
$$

Step 2: Add the equations (5), (6) as well as equations (7), (8) to obtain the following equations, respectively.

$$
{ }^{c} D^{\alpha} \psi_{r}(t)=a \psi_{r}(t)+2 b
$$

where,

$$
\psi_{r}(t)=\underline{y_{r}}(t)+\bar{y}_{r}(t)
$$

So,

$$
\psi_{r}(0)=S_{1}+S_{3}+r\left(2 S_{2}-S_{1}-S_{3}\right) .
$$

Step 3: Finding a solution to the Eq. (9) by applying FNT and the properties of Mittag-Leffler function. 
Step 4: Subtract the equations (5), (6) as well as equations (7), (8) to obtain the following equations, respectively

$$
{ }^{C} D^{\alpha} \phi_{r}(t)=a \phi_{r}(t)
$$

where,

$$
\phi_{r}(t)=\underline{y}_{r}(t)-\bar{y}_{r}(t)
$$

So,

$$
\phi_{r}(0)=S_{1}-S_{3}+r\left(S_{3}-S_{1}\right) .
$$

Step 5: Getting the solution to the Eq.(12) by applying FNT and the properties of Mittag-Leffler function.

Step 6: Return to step 1 and step 2, such that Firstly: adding the equations (10), (13) to get the lower bound of solution $\underline{y}_{r}(t)$. Secondly: subtracting the same equations to get the upper bound of solution $\bar{y}_{r}(t)$.

\section{The Solutions of FFODEs}

In this section, the solutions of FFODEs (3)-(4) will be based on the method steps mentioned above in the algorithm through two subsections depending on the orders of Caputo's H-difference ${ }^{C} D^{\alpha}$. First: includes the solutions when the order is $0<\alpha \leq 1$ and Second: includes other solutions when the order is $1<\alpha \leq 2$.

\subsection{The solutions of FFODEs of the order $0<\alpha \leq 1$}

Now, the FFODEs (3)-(4) of order $\alpha \in(0,1]$ can be solved through two cases depending on the signal of the constant $a$.

Case i: Let $a>0$, then the solutions of (3)-(4) can be found by applying the (i) and (ii) forms of Theorem 2.11, as follows:

First Form: Assume that $[\tilde{y}(t)]^{r}$ is Caputo's H-difference function in (1) -differentiable, from Theorem 2.11 (i), then the solution is

$$
\begin{aligned}
& \underline{y}_{r}(t)=\left\{S_{1}+r\left(S_{2}-S_{1}\right)\right\} E_{\alpha}\left(a t^{\alpha}\right)+b t^{\alpha} E_{\alpha, \alpha+1}\left(a t^{\alpha}\right), \\
& \bar{y}_{r}(t)=\left\{S_{3}+r\left(S_{2}-S_{3}\right)\right\} E_{\alpha}\left(a t^{\alpha}\right)+b t^{\alpha} E_{\alpha, \alpha+1}\left(a t^{\alpha}\right) .
\end{aligned}
$$

Second Form: Assume that $[\tilde{y}(t)]^{r}$ is Caputo's H-difference function in (2) -differentiable, then from Theorem 2.11 (ii), then the solution is 


$$
\begin{aligned}
\underline{y_{r}}(t)=\frac{1}{2}\left\{S_{1}+\right. & \left.S_{3}+r\left(2 S_{2}-S_{1}-S_{3}\right)\right\} E_{\alpha}\left(a t^{\alpha}\right)+\frac{1}{2}\left\{S_{1}-S_{3}+r\left(S_{3}-S_{1}\right)\right\} E_{\alpha}\left(-a t^{\alpha}\right) \\
& +b t^{\alpha} E_{\alpha, \alpha+1}\left(a t^{\alpha}\right), \\
\bar{y}_{r}(t)=\frac{1}{2}\left\{S_{1}+\right. & \left.S_{3}+r\left(2 S_{2}-S_{1}-S_{3}\right)\right\} E_{\alpha}\left(a t^{\alpha}\right)-\frac{1}{2}\left\{S_{1}-S_{3}+r\left(S_{3}-S_{1}\right)\right\} E_{\alpha}\left(-a t^{\alpha}\right) \\
& +b t^{\alpha} E_{\alpha, \alpha+1}\left(a t^{\alpha}\right) .
\end{aligned}
$$

Case ii: Let $a<0$, then (3)-(4) becomes

$$
\begin{gathered}
{\left[{ }^{c} D^{\alpha} \underline{y}_{r}(t),{ }^{c} D^{\alpha} \bar{y}_{r}(t)\right]=a\left[\bar{y}_{r}(t), \underline{y}_{r}(t)\right]+b,} \\
{\left[\underline{y}_{r}(0), \bar{y}_{r}(0)\right]=\left[S_{1}+r\left(S_{2}-S_{1}\right), S_{3}-r\left(S_{3}-S_{2}\right)\right] .}
\end{gathered}
$$

In order to get the solutions of the above equations. By applying the (i) and (ii) forms of Theorem 2.11, as follows:

First form: Consider that $[\tilde{y}(t)]^{r}$ is Caputo's H-difference function in (1) -differentiable, from Theorem 2.11 (i). Note that getting the same lower and upper bound for a second form of Case i.

Second form: Assume that $[\tilde{y}(t)]^{r}$ is Caputo's H-difference function in (2) -differentiable, then from Theorem 2.11 (ii). Note that the same lower and upper bound are getting to the first form of Case $i$.

\subsection{The solutions of FFODEs of the order $1<\alpha \leq 2$}

If the FFODEs (3) - (4) are of the order $\alpha \in(1,2]$, then they are also solved using the same algorithm of the method and these equations take the form:

$$
\left[{ }^{c} D^{\alpha} \underline{y}_{r}(t),{ }^{c} D^{\alpha} \bar{y}_{r}(t)\right]=a\left[\underline{y}_{r}(t), \bar{y}_{r}(t)\right]+b,
$$

subject to the (FIC) $[\tilde{y}(0)]^{r}=\delta^{r},\left[\tilde{y}_{t}(0)\right]^{r}=\varepsilon^{r}$. Put $\delta^{r}=\varepsilon^{r}$, then,

$$
\begin{aligned}
{\left[\tilde{y}_{t}(0)\right]^{r} } & =[\tilde{y}(0)]^{r}=\left[S_{1}, S_{2}, S_{3}\right], \\
& =\left[S_{1}+r\left(S_{2}-S_{1}\right), S_{3}-r\left(S_{3}-S_{2}\right)\right] .
\end{aligned}
$$

The above equation will be solved in two cases depending on the signal of the constant $a$.

Case j: Let $a>0$, finding the solutions of (15)-(16) by applying the (iii), (iv), (v) and (vi) forms of Theorem 2.11, respectively as in the following the systems. 
(1-1) System: Assume that $[\tilde{y}(t)]^{r}$ is Caputo's H-difference function $(1,1)$-differentiable, from Theorem2.11 (iii), then the solution of (15)-(16) is

$$
\begin{gathered}
\underline{y}_{r}(t)=\left\{S_{1}+r\left(S_{2}-S_{1}\right)\right\}\left(E_{\alpha}\left(a t^{\alpha}\right)+t E_{\alpha, 2}\left(a t^{\alpha}\right)\right)+b t^{\alpha} E_{\alpha, \alpha+1}\left(a t^{\alpha}\right), \\
\bar{y}_{r}(t)=\left\{S_{3}+r\left(S_{2}-S_{3}\right)\right\}\left(E_{\alpha}\left(a t^{\alpha}\right)+t E_{\alpha, 2}\left(a t^{\alpha}\right)\right)+b t^{\alpha} E_{\alpha, \alpha+1}\left(a t^{\alpha}\right) .
\end{gathered}
$$

(1-2) System: Suppose that $[\tilde{y}(t)]^{r}$ is Caputo's H-difference function in $(1,2)$-differentiable, from Theorem 2.11 (iv), note that (15)-(16) becomes

$$
\left[{ }^{c} D^{\alpha} \bar{y}_{r}(t),{ }^{c} D^{\alpha} \underline{y r}_{r}(t)\right]=a\left[\underline{y}_{r}(t), \bar{y}_{r}(t)\right]+b,
$$

and FIC is

$$
\begin{array}{cc}
\underline{y}_{r}(0)=\underline{\delta}^{r}, & \bar{y}_{r}(0)=\bar{\delta}^{r}, \\
\underline{y}_{t r}(0)=\underline{\varepsilon}^{r}, & \bar{y}_{t r}(0)=\bar{\varepsilon}^{r} .
\end{array}
$$

Then the solution is

$$
\begin{aligned}
\underline{y_{r}}(t)=\frac{1}{2}\left\{S_{1}+\right. & \left.S_{3}+r\left(2 S_{2}-S_{1}-S_{3}\right)\right\}\left(E_{\alpha}\left(a t^{\alpha}\right)+t E_{\alpha, 2}\left(a t^{\alpha}\right)\right)+\frac{1}{2}\left\{S_{1}-S_{3}\right. \\
& \left.+r\left(S_{3}-S_{1}\right)\right\}\left(E_{\alpha}\left(-a t^{\alpha}\right)+t E_{\alpha, 2}\left(-a t^{\alpha}\right)\right)+b t^{\alpha} E_{\alpha, \alpha+1}\left(a t^{\alpha}\right), \\
\bar{y}_{r}(t)=\frac{1}{2}\left\{S_{1}+\right. & \left.S_{3}+r\left(2 S_{2}-S_{1}-S_{3}\right)\right\}\left(E_{\alpha}\left(a t^{\alpha}\right)+t E_{\alpha, 2}\left(a t^{\alpha}\right)\right)+\frac{1}{2}\left\{S_{1}-S_{3}\right. \\
& \left.+r\left(S_{3}-S_{1}\right)\right\}\left(E_{\alpha}\left(-a t^{\alpha}\right)+t E_{\alpha, 2}\left(-a t^{\alpha}\right)\right)+b t^{\alpha} E_{\alpha, \alpha+1}\left(a t^{\alpha}\right) .
\end{aligned}
$$

(2-1) System: Consider that $[\tilde{y}(t)]^{r}$ is Caputo's H-difference function in $(2,1)$-differentiable, from Theorem $2.11(\mathrm{v})$,

Note that (15)-(16) becomes

$$
\left[{ }^{c} D^{\alpha} \bar{y}_{r}(t),{ }^{c} D^{\alpha} \underline{y}_{r}(t)\right]=a\left[\underline{y}_{r}(t), \bar{y}_{r}(t)\right]+b,
$$

and FIC is

$$
\begin{aligned}
& \underline{y}_{r}(0)=\underline{\delta}^{r}, \quad \bar{y}_{r}(0)=\bar{\delta}^{r}, \\
& \underline{y}_{t r}(0)=\bar{\varepsilon}^{r}, \bar{y}_{t r}(0)=\underline{\varepsilon}^{r} .
\end{aligned}
$$

Then the solution is

$$
\begin{aligned}
\underline{y_{r}}(t)=\frac{1}{2}\left\{S_{1}+\right. & \left.S_{3}+r\left(2 S_{2}-S_{1}-S_{3}\right)\right\}\left(E_{\alpha}\left(a t^{\alpha}\right)+t E_{\alpha, 2}\left(a t^{\alpha}\right)\right)+\frac{1}{2}\left\{S_{1}-S_{3}\right. \\
& \left.+r\left(S_{3}-S_{1}\right)\right\}\left(E_{\alpha}\left(-a t^{\alpha}\right)-t E_{\alpha, 2}\left(-a t^{\alpha}\right)\right)+b t^{\alpha} E_{\alpha, \alpha+1}\left(a t^{\alpha}\right),
\end{aligned}
$$




$$
\begin{aligned}
\bar{y}_{r}(t)=\frac{1}{2}\left\{S_{1}+\right. & \left.S_{3}+r\left(2 S_{2}-S_{1}-S_{3}\right)\right\}\left(E_{\alpha}\left(a t^{\alpha}\right)+t E_{\alpha, 2}\left(a t^{\alpha}\right)\right)-\frac{1}{2}\left\{S_{1}-S_{3}\right. \\
& \left.+r\left(S_{3}-S_{1}\right)\right\}\left(E_{\alpha}\left(-a t^{\alpha}\right)-t E_{\alpha, 2}\left(-a t^{\alpha}\right)\right)+b t^{\alpha} E_{\alpha, \alpha+1}\left(a t^{\alpha}\right) .
\end{aligned}
$$

(2-2) System: Assume that $[\tilde{y}(t)]^{r}$ is Caputo's H-difference function in $(2,2)$-differentiable, from Theorem 2.11 (vi),

Note that (15)-(16) becomes

$$
\left[{ }^{c} D^{\alpha} \underline{y}_{r}(t),{ }^{c} D^{\alpha} \bar{y}_{r}(t)\right]=a\left[\underline{y}_{r}(t), \bar{y}_{r}(t)\right]+b,
$$

and FIC is

$$
\begin{aligned}
& \underline{y}_{r}(0)=\underline{\delta}^{r}, \quad \bar{y}_{r}(0)=\bar{\delta}^{r}, \\
& \underline{y}_{t r}(0)=\bar{\varepsilon}^{r}, \quad \bar{y}_{t r}(0)=\underline{\varepsilon}^{r} .
\end{aligned}
$$

Then the solution is

$$
\begin{gathered}
\underline{y_{r}}(t)=\left\{S_{1}+r\left(S_{2}-S_{1}\right)\right\} E_{\alpha}\left(a t^{\alpha}\right)+\left\{S_{3}+r\left(S_{2}-S_{3}\right)\right\} t E_{\alpha, 2}\left(a t^{\alpha}\right)+b t^{\alpha} E_{\alpha, \alpha+1}\left(a t^{\alpha}\right), \\
\bar{y}_{r}(t)=\left\{S_{3}+r\left(S_{2}-S_{3}\right)\right\} E_{\alpha}\left(a t^{\alpha}\right)+\left\{S_{1}+r\left(S_{2}-S_{1}\right)\right\} t E_{\alpha, 2}\left(a t^{\alpha}\right) \\
+b t^{\alpha} E_{\alpha, \alpha+1}\left(a t^{\alpha}\right) .
\end{gathered}
$$

Case jj: Let $a<0$, then (15)-(16) take this form

$$
\left[{ }^{c} D^{\alpha} \underline{y}_{r}(t),{ }^{c} D^{\alpha} \bar{y}_{r}(t)\right]=a\left[\bar{y}_{r}(t), \underline{y}_{r}(t)\right]+b,
$$

subject to the $\operatorname{FIC}[\tilde{y}(0)]^{r}=\delta^{r},\left[\tilde{y}_{t}(0)\right]^{r}=\varepsilon^{r}$. Put $\delta^{r}=\varepsilon^{r}$, then,

$$
\begin{gathered}
\quad\left[\tilde{y}_{t}(0)\right]^{r}=[\tilde{y}(0)]^{r}=\left[S_{1}, S_{2}, S_{3}\right], \\
=\left[S_{1}+r\left(S_{2}-S_{1}\right), S_{3}-r\left(S_{3}-S_{2}\right)\right] .
\end{gathered}
$$

Now, finding the solutions of (17)-(18). By applying the (iii), (iv), (v) and (vi) forms of Theorem 2.11, respectively as in the following systems:

(1-1) System: Consider that $[\tilde{y}(t)]^{r}$ is Caputo's H-difference function in $(1,1)$-differentiable, from Theorem 2.11 (iii), then the solution of (17)-(18) is

$$
\begin{aligned}
\underline{y}_{r}(t)=\frac{1}{2}\left\{S_{1}+\right. & \left.S_{3}+r\left(2 S_{2}-S_{1}-S_{3}\right)\right\}\left(E_{\alpha}\left(a t^{\alpha}\right)+t E_{\alpha, 2}\left(a t^{\alpha}\right)\right)+\frac{1}{2}\left\{S_{1}-S_{3}\right. \\
& \left.+r\left(S_{3}-S_{1}\right)\right\}\left(E_{\alpha}\left(-a t^{\alpha}\right)+t E_{\alpha, 2}\left(-a t^{\alpha}\right)\right)+b t^{\alpha} E_{\alpha, \alpha+1}\left(a t^{\alpha}\right),
\end{aligned}
$$




$$
\begin{aligned}
\bar{y}_{r}(t)=\frac{1}{2}\left\{S_{1}+\right. & \left.S_{3}+r\left(2 S_{2}-S_{1}-S_{3}\right)\right\}\left(E_{\alpha}\left(a t^{\alpha}\right)+t E_{\alpha, 2}\left(a t^{\alpha}\right)\right)-\frac{1}{2}\left\{S_{1}-S_{3}\right. \\
& \left.+r\left(S_{3}-S_{1}\right)\right\}\left(E_{\alpha}\left(-a t^{\alpha}\right)+t E_{\alpha, 2}\left(-a t^{\alpha}\right)\right)+b t^{\alpha} E_{\alpha, \alpha+1}\left(a t^{\alpha}\right) .
\end{aligned}
$$

(1-2) System: Suppose that $[\tilde{y}(t)]^{r}$ is Caputo's H-difference function in $(1,2)$-differentiable, from Theorem 2.11 (iv),

Note that (17)-(18) becomes

$$
\left[{ }^{c} D^{\alpha} \bar{y}_{r}(t),{ }^{c} D^{\alpha} \underline{y}_{r}(t)\right]=a\left[\bar{y}_{r}(t), \underline{y}_{r}(t)\right]+b,
$$

and FIC is

$$
\begin{aligned}
& \underline{y}_{r}(0)=\underline{\delta}^{r}, \quad \bar{y}_{r}(0)=\bar{\delta}^{r}, \\
& \underline{y}_{t r}(0)=\underline{\varepsilon}^{r}, \quad \bar{y}_{t r}(0)=\bar{\varepsilon}^{r} .
\end{aligned}
$$

After solving this system, obtaining the same results in (1-1) system for the Case j.

(2-1) System: Assume that $[\tilde{y}(t)]^{r}$ is Caputo's H-difference function in $(2,1)$-differentiable, from Theorem 2.11 (v),

Note that (17)-(18) becomes

$$
\left[{ }^{c} D^{\alpha} \bar{y}_{r}(t),{ }^{c} D^{\alpha} \underline{y r}_{r}(t)\right]=a\left[\bar{y}_{r}(t), \underline{y_{r}}(t)\right]+b,
$$

and (FIC) is

$$
\begin{array}{cc}
\underline{y}_{r}(0)=\underline{\delta}^{r}, & \bar{y}_{r}(0)=\bar{\delta}^{r}, \\
\underline{y}_{t r}(0)=\bar{\varepsilon}^{r}, & \bar{y}_{t r}(0)=\underline{\varepsilon}^{r} .
\end{array}
$$

After solving this system obtaining the same results in (2-2) system for the Case j.

(2-2) System: Assume that $[\tilde{y}(t)]^{r}$ is Caputo's H-difference function in $(2,2)$-differentiable, from Theorem 2.11 (vi),

Note that (17)-(18) becomes

$$
\left[{ }^{c} D^{\alpha} \underline{y}_{r}(t),{ }^{c} D^{\alpha} \bar{y}_{r}(t)\right]=a\left[\bar{y}_{r}(t), \underline{y_{r}}(t)\right]+b,
$$

and (FIC) is

$$
\underline{y}_{r}(0)=\underline{\delta}^{r}, \quad \bar{y}_{r}(0)=\bar{\delta}^{r},
$$




$$
\underline{y}_{t r}(0)=\bar{\varepsilon}^{r}, \quad \bar{y}_{t r}(0)=\underline{\varepsilon}^{r} .
$$

Then the solution is

$$
\begin{aligned}
\underline{y_{r}}(t)=\left\{\frac { 1 } { 2 } \left\{S_{1}+\right.\right. & \left.S_{3}+r\left(2 S_{2}-S_{1}-S_{3}\right)\right\}\left(E_{\alpha}\left(a t^{\alpha}\right)+t E_{\alpha, 2}\left(a t^{\alpha}\right)\right) \\
& +\frac{1}{2}\left\{S_{1}-S_{3}+r\left(S_{3}-S_{1}\right)\right\}\left(E_{\alpha}\left(-a t^{\alpha}\right)-t E_{\alpha, 2}\left(-a t^{\alpha}\right)\right) \\
& +b t^{\alpha} E_{\alpha, \alpha+1}\left(a t^{\alpha}\right), \\
\bar{y}_{r}(t)=\frac{1}{2}\left\{S_{1}+\right. & \left.S_{3}+r\left(2 S_{2}-S_{1}-S_{3}\right)\right\}\left(E_{\alpha}\left(a t^{\alpha}\right)+t E_{\alpha, 2}\left(a t^{\alpha}\right)\right) \\
& -\frac{1}{2}\left\{S_{1}-S_{3}+r\left(S_{3}-S_{1}\right)\right\}\left(E_{\alpha}\left(-a t^{\alpha}\right)-t E_{\alpha, 2}\left(-a t^{\alpha}\right)\right) \\
& +b t^{\alpha} E_{\alpha, \alpha+1}\left(a t^{\alpha}\right) .
\end{aligned}
$$

\section{Main Results and Discussion}

In this section, assume that the FIC is $\left[\tilde{y}_{t}(0)\right]^{r}=[\tilde{y}(0)]^{r}=[1,2,3]$. Taking $a=b=$ 1 for Cases i and j so, $a=-1, b=1$ for Cases ii and jj. Firstly, the numerical solutions of (3)-(4) for Cases i and ii are listed in Tables 1 and 2, respectively. For graphical results, please see in Figures 1 and 2.

Table 1: Numerical solutions at Case i; First and Second forms of FFODEs (3)-(4) for $\alpha=0.5$.

\begin{tabular}{lcccc}
\hline & \multicolumn{2}{c}{ Case i; First form $\alpha=0.5$} & \multicolumn{2}{c}{ Case i; Second form $\alpha=0.5$} \\
\hline$r$ & $\underline{y}$ & $\bar{y}$ & $\underline{y}$ & $\bar{y}$ \\
\hline 0 & 27.3517 & 55.5461 & 41.0128 & 41.8850 \\
0.1 & 28.7614 & 54.1364 & 41.0564 & 41.8414 \\
0.2 & 30.1711 & 52.7267 & 41.1000 & 41.7978 \\
0.3 & 31.5808 & 51.3170 & 41.1436 & 41.7542 \\
0.4 & 32.9906 & 49.9072 & 41.1872 & 41.7106 \\
0.5 & 34.4003 & 48.4975 & 41.2308 & 41.6669 \\
0.6 & 35.8100 & 47.0878 & 41.2745 & 41.6233 \\
0.7 & 37.2197 & 45.6781 & 41.3181 & 41.5797 \\
0.8 & 38.6295 & 44.2683 & 41.3617 & 41.5361 \\
0.9 & 40.0392 & 42.8586 & 41.4053 & 41.4925 \\
1.0 & 41.4489 & 41.4489 & 41.4489 & 41.4489 \\
\hline
\end{tabular}



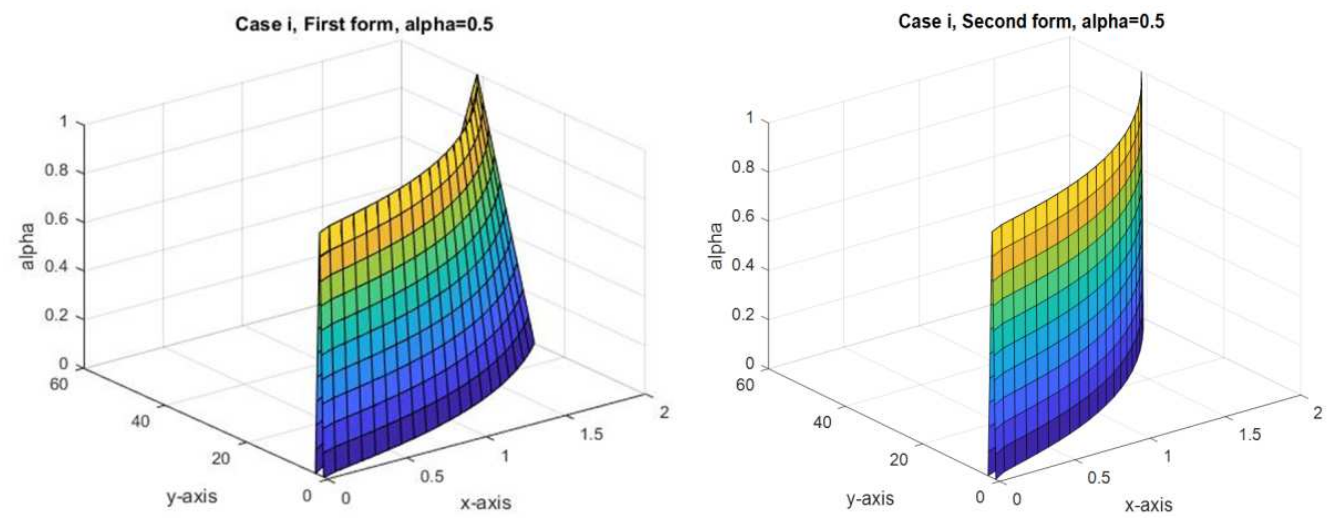

Figure 1: The numerical solutions at Case i; First and Second forms of FFODEs (3)-(4) for $\alpha=0.5$.

Table 2: Numerical solutions at Case ii; First and Second forms of FFODEs (3)-(4) for $\alpha=0.5$.

Case i; Second form $\alpha=0.5$

\begin{tabular}{lcccc}
\hline$r$ & $\underline{y}$ & $\bar{y}$ & $\underline{y}$ & $\bar{y}$ \\
\hline 0 & -12.5039 & 15.6905 & 1.1572 & 2.0294 \\
0.1 & -11.0942 & 14.2808 & 1.2008 & 1.9858 \\
0.2 & -9.6845 & 12.8711 & 1.2444 & 1.9422 \\
0.3 & -8.2748 & 11.4614 & 1.2880 & 1.8986 \\
0.4 & -6.8650 & 10.0516 & 1.3316 & 1.8550 \\
0.5 & -5.4553 & 8.6419 & 1.3752 & 1.8113 \\
0.6 & -4.0456 & 7.2322 & 1.4189 & 1.7677 \\
0.7 & -2.6359 & 5.8225 & 1.4625 & 1.7241 \\
0.8 & -1.2261 & 4.4127 & 1.5061 & 1.6805 \\
0.9 & 0.1836 & 3.0030 & 1.5497 & 1.6369 \\
1.0 & 1.5933 & 1.5933 & 1.5933 & 1.5933 \\
\hline
\end{tabular}



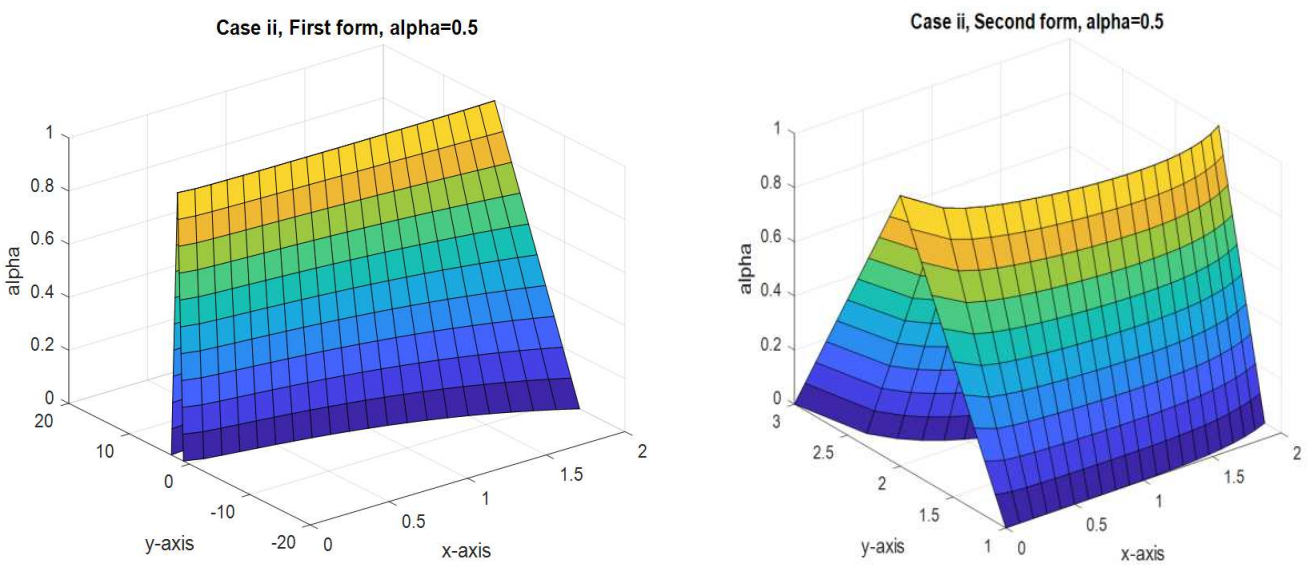

Figure 2: The numerical solutions at Case ii; First and Second forms of FFODEs (3)-(4) for $\alpha=0.5$.

Secondly, the numerical solutions of (13)-(14) for Cases $\mathrm{j}$ and $\mathrm{jj}$ are listed in Tables 3, 4, 5 and 6, respectively. For graphical results, please see in Figures 3 and 4. Numerical solutions for all cases are got by extending Mittag-Leffler functions up to 10 terms.

Table 3: Numerical solutions at Case j; (1-1) and (1-2) systems of FFODEs (13)-(14) for $\alpha=1.3$.

\begin{tabular}{lcccc}
\hline & \multicolumn{2}{c}{ Case j; $(1-1)$ system, $\alpha=1.3$} & \multicolumn{2}{c}{ Case j; (1-2) system, $\alpha=1.3$} \\
\hline$r$ & $\underline{y}$ & $\bar{y}$ & $\underline{y}$ & $\bar{y}$ \\
\hline 0 & 15.5994 & 37.3200 & 25.6504 & 27.3203 \\
0.1 & 16.6854 & 36.2340 & 25.7313 & 27.2343 \\
0.2 & 17.7714 & 35.1479 & 25.8122 & 27.1482 \\
0.3 & 18.8575 & 34.0619 & 25.8932 & 27.0621 \\
0.4 & 19.9435 & 32.9759 & 25.9741 & 26.9761 \\
0.5 & 21.0295 & 31.8899 & 26.0550 & 26.8900 \\
0.6 & 22.1156 & 30.8038 & 26.1360 & 26.8040 \\
0.7 & 23.2016 & 29.7178 & 26.2169 & 26.7179 \\
0.8 & 24.2876 & 28.6318 & 26.2978 & 26.6318 \\
0.9 & 25.3737 & 27.5457 & 26.3788 & 26.5458 \\
1.0 & 26.4597 & 26.4597 & 26.4597 & 26.4597 \\
\hline
\end{tabular}


Table 4: numerical solutions at Case j; (2-1) and (2-2) systems of FFODEs (13)-(14) for $\alpha=1.3$.

\begin{tabular}{lcccc}
\hline & Case $\mathrm{j} ;(2-1)$ system, $\alpha=1.3$ & \multicolumn{2}{c}{ Case $\mathrm{j} ;(2-2)$ system, $\alpha=1.3$} \\
\hline$r$ & $\underline{y}$ & $\bar{y}$ & $\underline{y}$ & $\bar{y}$ \\
\hline 0 & 25.5991 & 27.3203 & 25.8419 & 27.0775 \\
0.1 & 25.6851 & 27.2343 & 25.9037 & 27.0157 \\
0.2 & 25.7712 & 27.1482 & 25.9654 & 26.9539 \\
0.3 & 25.8572 & 27.0621 & 26.0272 & 26.8922 \\
0.4 & 25.9433 & 26.9761 & 26.0890 & 26.8304 \\
0.5 & 26.0294 & 26.8900 & 26.1508 & 26.7686 \\
0.6 & 26.1154 & 26.8040 & 26.2126 & 26.7068 \\
0.7 & 26.2015 & 26.7179 & 26.2744 & 26.6450 \\
0.8 & 26.2876 & 26.6318 & 26.3361 & 26.5833 \\
0.9 & 26.3736 & 26.5458 & 26.3979 & 26.5215 \\
1.0 & 26.4597 & 26.4597 & 26.4597 & 26.4597 \\
\hline
\end{tabular}
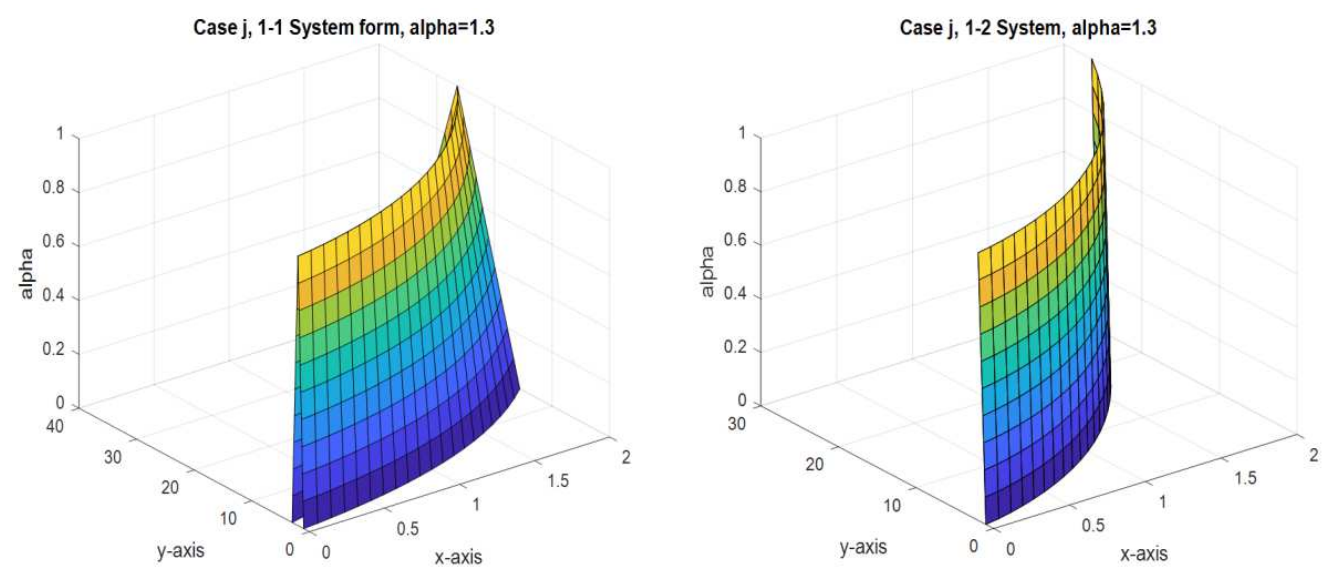

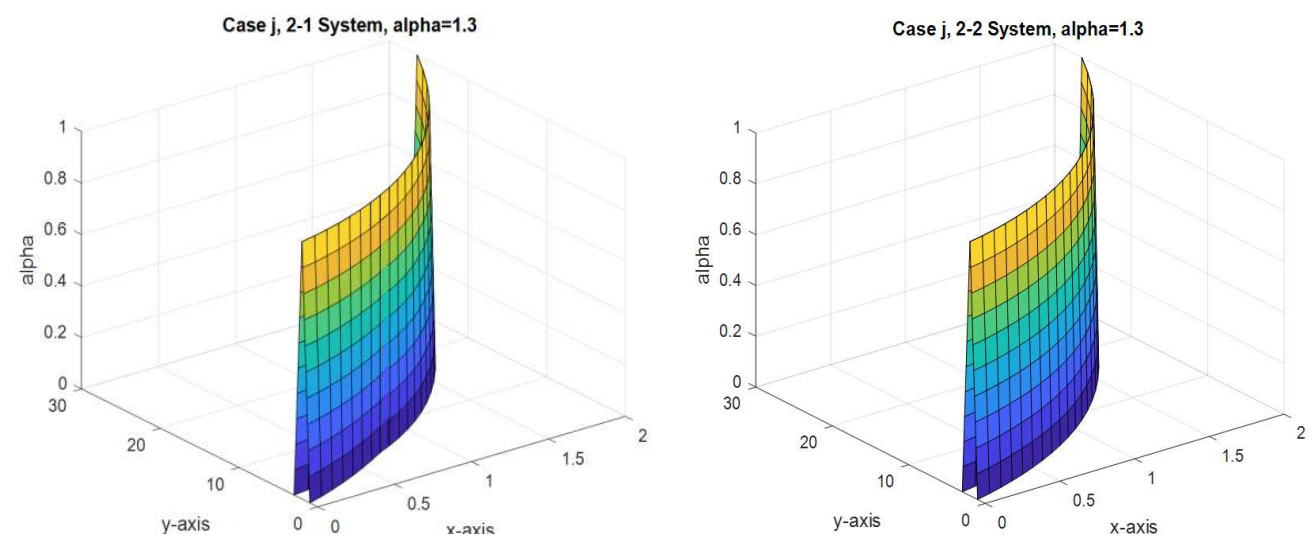

Figures 3: The numerical solutions at Case $\mathbf{j}$; (1-1),(1-2),(2-1) and (2-2) systems of FFODEs (13)-(14) for $\alpha=1.3$.

Table 5: Numerical solutions at Case jj; (1-1) and (1-2) systems of FFODEs (13)-(14) for $\alpha=1.3$.

\begin{tabular}{ccccc}
\hline & Case $\mathbf{j j} ;(1-1)$ & system, $\alpha=1.3$ & \multicolumn{2}{c}{ Case $\mathbf{j j} ;(1-2)$ system, $\alpha=1.3$} \\
\hline$r$ & $\underline{y}$ & $\bar{y}$ & $\underline{y}$ & $\bar{y}$ \\
\hline 0 & -8.2160 & 3.2621 & 1.8350 & 3.4536 \\
0.1 & -7.1300 & 3.2003 & 1.9159 & 3.3727 \\
0.2 & -6.0440 & 3.1385 & 1.9968 & 3.2917 \\
0.3 & -4.9579 & 3.0767 & 2.0778 & 3.2108 \\
0.4 & -3.8719 & 3.0150 & 2.1587 & 3.1299 \\
0.5 & -2.7859 & 2.9532 & 2.2396 & 3.0489 \\
0.6 & -1.6998 & 2.8914 & 2.3206 & 2.9680 \\
0.7 & -0.6138 & 2.8296 & 2.4015 & 2.8871 \\
0.8 & 0.4722 & 2.7678 & 2.4824 & 2.8061 \\
0.9 & 1.5582 & 2.7061 & 2.5634 & 2.7252 \\
1.0 & 2.6443 & 2.6443 & 2.6443 & 2.6443 \\
\hline
\end{tabular}


Table 6: Numerical solutions at Case $\mathbf{j j}$; (2-1) and (2-2) systems of FFODEs (13)-(14) for $\alpha=1.3$.

\begin{tabular}{ccccc}
\hline & Case $\mathbf{j j} ;(2-1)$ & system, $\alpha=1.3$ & \multicolumn{2}{c}{ Case $\mathbf{j j} ;(2-2)$ system, $\alpha=1.3$} \\
\hline$r$ & $\underline{y}$ & $\bar{y}$ & $\underline{y}$ & $\bar{y}$ \\
\hline 0 & 3.5049 & 1.7836 & 2.0265 & 3.2621 \\
0.1 & 3.4189 & 1.8697 & 2.0883 & 3.2003 \\
0.2 & 3.3328 & 1.9558 & 2.1500 & 3.1385 \\
0.3 & 3.2467 & 2.0418 & 2.2118 & 3.0767 \\
0.4 & 3.1607 & 2.1279 & 2.2736 & 3.0150 \\
0.5 & 3.0746 & 2.2140 & 2.3354 & 2.9532 \\
0.6 & 2.9885 & 2.3000 & 2.3972 & 2.8914 \\
0.7 & 2.9025 & 2.3861 & 2.4589 & 2.8296 \\
0.8 & 2.8164 & 2.4722 & 2.5207 & 2.7678 \\
0.9 & 2.7303 & 2.5582 & 2.5825 & 2.7061 \\
1.0 & 2.6443 & 2.6443 & 2.6443 & 2.6443 \\
\hline
\end{tabular}
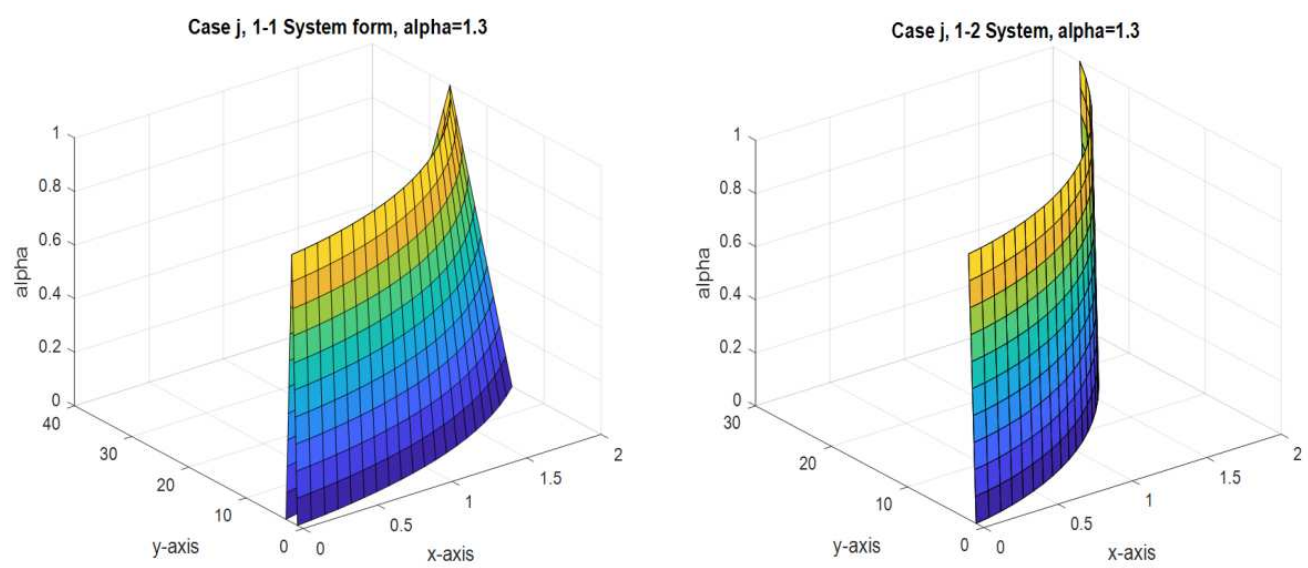

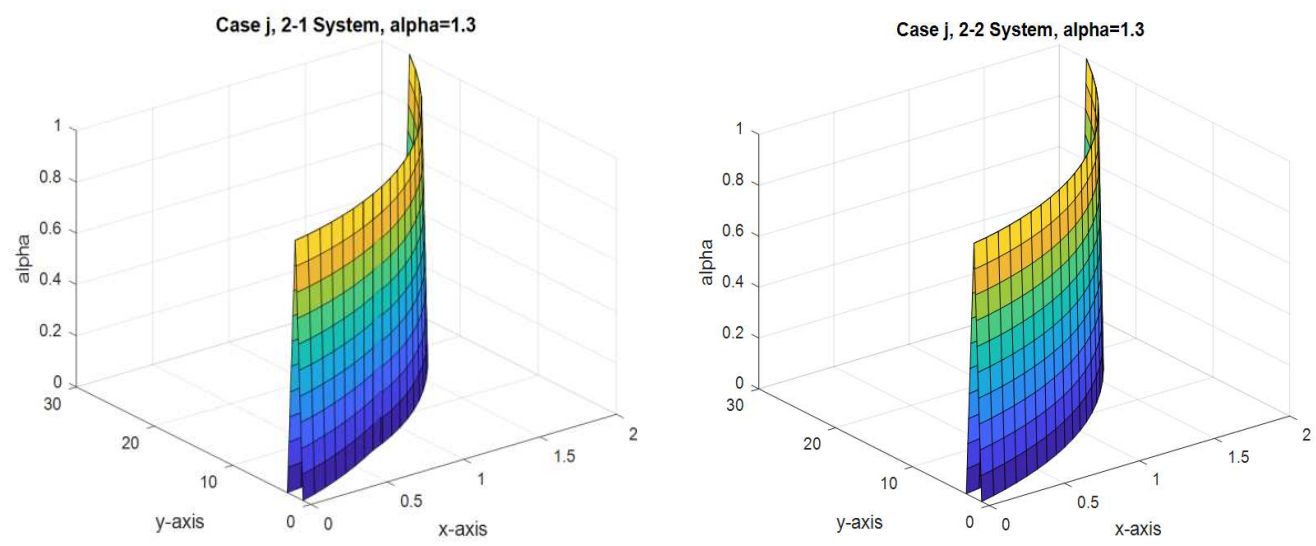

Figure 4: The numerical solutions at Case $\mathbf{j j}$; (1-1), (1-2), (2-1) and (2-2) systems of FFODEs (13)-(14) for $\alpha=1.3$.

\section{Conclusions}

In the present paper, we obtained the solutions of FFODEs involving fuzzy Caputo's H-difference according to the method proposed by [12]. A new property of fuzzy natural transform for fuzzy Caputo's H-difference has been introduced. The new property has been used to establish a procure for solving linear FFODEs. These equations have been solved numerically to show that FNT is functional. For future research, we contemplate to apply FNT on nonlinear fuzzy differential equations of fractional order. Finally, we demonstrated that the proposed approach has good effectiveness and accuracy to compute the fuzzy solution set and for applications to FFODEs.

\section{References}

[1] N. A. Abdul Rahman and M. Z. Ahmad, Solving fuzzy fractional differential equations using fuzzy Sumudu transform, Journal of Nonlinear Sciences and Applications 10(1) (2017), 2620-2632. https://doi.org/10.22436/jnsa.010.05.28

[2] O. Abu Arqub and M. Al-Smadi, Fuzzy conformable fractional differential equations: novel extended approach and new numerical solutions, Methodologies and Application 24(16) (2020), 12501-12522. https://doi.org/10.1007/s00500-020-04687-0

[3] R. P. Agarwal, V. Lakshmikantham and J. J. Nieto, On the concept of solution for fractional differential equations with uncertainty, Nonlinear Analysis 72(6) (2010), 28592862. https://doi.org/10.1016/j.na.2009.11.029 
[4] M. Alaroud, M. Al-Smadi, R. R. Ahmad and U. K. Salma, An analytical numerical method for solving fuzzy fractional Volterra integro-differential equations, Symmetry 2(11) (2019), 1-19. https://doi.org/10.3390/sym11020205

[5] T. Allahviranloo, A. Armand and Z. Gouyandeh, Fuzzy fractional differential equations under generalized fuzzy Caputo derivative, Journal of Intelligent \& Fuzzy Systems 26(3) (2014), 1481-1490. https://doi.org/10.3233/IFS-130831

[6] M. Alshammari, M. Al-Smadi, O. Abu Arqub, I. Hashim and M. Alias, Residual series representation algorithm for solving fuzzy Duffing oscillator equations, Symmetry 12(4) (2020), 1-20. https://doi.org/10.3390/sym12040572

[7] A. A. Attiya, Some applications of Mittag-Leffler function in the unit disk, Faculty of Sciences and Mathematics 30(7) (2016), 2075-2081. https://doi.org/10.2298/FIL1607075A

[8] S. K. Al-Omari and S. Araci, Certain fundamental properties of generalized natural transform in generalized spaces, Advances in Difference Equations 1(163) (2021), 1-11. https://doi.org/10.1186/s13662-021-03328-6

[9] B. Bede, I. J. Rudas and A. L. Bencsik, First order linear fuzzy differential equations under generalized differentiability, Information Sciences 177(7) (2007), 1648-1662. https://doi.org/10.1016/j.ins.2006.08.021

[10] F. B. M. Belgacem and R. Silambarasan, Theory of natural transform, Mathematics in Engineering, Science and Aerospace 3(1) (2012), 105-135. https://doi.org/10.1063/1.4765477

[11] A. H. Bhrawy and M. A. Zaky, Shifted fractional-order Jacobi orthogonal functions: application to a system of fractional differential equations, App. Math. Mod. 40(1) (2016), 832-845. https://doi.org/10.1016/j.apm.2015.06.012

[12] A. K. Das and T. K. Roy, Exact solution of some linear fuzzy fractional differential equation using Laplace transform method, Global Journal of Pure and Applied Mathematics 13(9) (2017), 5427-5435.

[13] V. P. Dubey, R. Kumar, D. Kumar, I. Khan and J. Singh, An efficient computational scheme for nonlinear time fractional systems of partial differential equations arising in physical sciences, Advances in Difference Equations 46(1) (2020), 1-27.

https://doi.org/10.1186/s13662-020-2505-6

[14] M. Friedman, M. Ming and A. Kandel, Numerical solution of fuzzy differential and integral equations, Fuzzy Sets and Systems 106(1) (1999), 35-48.

https://doi.org/10.1016/S0165-0114(98)00355-8 
[15] M. Friedman, M. Ming and A. Kandel, Fuzzy linear systems, Fuzzy Sets and Systems 96(2) (1998), 201-209. https://doi.org/10.1016/S0165-0114(96)00270-9

[16] A. Guezane-Lakoud and A. Kılıçman, On resonant mixed Caputo fractional differential equations, Boundary Value Problems 168(1) (2020), 1-13.

https://doi.org/10.1186/s13661-020-01465-7

[17] G. Gumah, M. Naser, M. Al-Smadi, S. Al-Omari and D. Baleanu, Numerical solutions of hybrid fuzzy differential equations in a Hilbert space, Applied Numerical Mathematics 151(1) (2020), 402-412. https://doi.org/10.1016/j.apnum.2020.01.008

[18] S. Hasan, M. Al-Smadi, A. El-Ajou, S. Momani, S. Hadid and Z. Al-Zhour, Numerical approach in the Hilbert space to solve a fuzzy Atangana-Baleanu fractional hybrid system, Chaos, Solitons \& Fractals 143(110506) (2021), 1-23.

https://doi.org/10.1016/j.chaos.2020.110506

[19] F. Hemati, M. Ghasemi and R. K. Ghaziani, Numerical solution of the multitier time-fractional diffusion equation based on reproducing kernel theory, Numerical Methods for Partial Differential Equations 37(1) (2020), 44-68.

https://doi.org/10.1002/num.22518

[20] L. Huang and Y. Bae, Chaotic dynamics of the fractional-love model with an external environment, Entropy 20(53) (2018), 1-14. https://doi.org/10.3390/e20010053

[21] L. Huang and Y. Bae, Nonlinear behavior in fractional-order Romeo and Juliet's love model influenced by external force with fuzzy function, International Journal of Fuzzy Systems 21(2) (2019), 630-638. https://doi.org/10.1007/s40815-018-0575-3

[22] H. Jafari, M. A. Firoozjaee and S. J. Johnston, An effective approach to solve a system fractional differential equation, Alexandria Engineering Journal 59(5) (2020), 3213-3219. https://doi.org/10.1016/j.aej.2020.08.015

[23] H. B. Kelishami, M. A. F. Araghi and M. Amirfakhrian, Applying the fuzzy CESTAC method to find the optimal shape parameter in solving fuzzy differential equations via RBF-Meshless methods, Soft Computing 24(83) (2020), 15655-15670. https://doi.org/10.1007/s00500-020-04890-z

[24] Z. H. Khan and W. A. Khan, N-transform. Properties and applications, NUST Journal of Engineering Sciences 1(1) (2008), 127-133.

[25] H. Khan, R Shah, P. Kumam and M. Arif, Analytical solutions of fractional-order heat and wave equations by the natural transform decomposition method, Entropy 21(597) (2019), 1-21. https://doi.org/10.3390/e21060597

[26] A. Khalouta and A. Kadem, Fractional natural decomposition method for solving a certain 
class of nonlinear time-fractional wave-like equations with variable coefficients, Acta Univ. Sapientiae Math. 11(1) (2019), 99-116. https://doi.org/10.2478/ausm-2019-0009

[27] A. Kılıçman and R. Silambarasan, Computing new solutions of algebro-geometric equation using the discrete inverse Sumudu transform. Advances in Difference Equations 323(1) (2018), 1-17. https://doi.org/10.1186/s13662-018-1785-6

[28] K. Köklü, Resolvent, natural, and Sumudu transformations: solution of logarithmic Kernel integral equations with natural transform, Mathematical Problems in Engineering 1(1) (2020), 1-7. https://doi.org/10.1155/2020/9746318

[29] M. Matusiak, Fast evaluation of Grünwald-Letnikov variable fractional-order differentiation and integration based on the FFT convolution, Advanced, Contemporary Control 1196(1) (2020), 879-890. https://doi.org/10.1007/978-3-030-50936-1_74

[30] M. Mazandarani and A. V. Kamyad, Modified fractional Euler method for solving fuzzy fractional initial value problem, Communications in Nonlinear Science and Numerical Simulation 18(1) (2013), 12-21. https://doi.org/10.1016/j.cnsns.2012.06.008

[31] G. D. Medina, N. R. Ojeda, J. H. Pereira and L. G. Romero, Fractional Laplace transform and fractional calculus, International Mathematical Forum 12(20) (2017), 991-1000. https://doi.org/10.12988/imf.2017.71194

[32] N. T. Negero, Zero-order Hankel transform method for partial differential equations, International Journal of Modern Sciences and Engineering Technology (IJMSET) 3(10) (2016), 24-36.

[33] V. Padmapriya, M. Kaliyappan and A. Manivannan, Numerical solutions of fuzzy fractional delay differential equations, International Journal of Fuzzy Logic and Intelligent Systems 20(3) (2020), 247-254. https://doi.org/10.5391/IJFIS.2020.20.3.247

[34] V. P. Priya and M. A Kaliyappan, Review of fuzzy fractional differential equations, International Journal of Pure and Applied Mathematics 110(10) (2017), 203-216.

[35] M. L. Puri and D. A. Ralescu, Differentials of fuzzy functions, Journal of Mathematics Analysis and Applications 91(2) (1983), 552-558.

https://doi.org/10.1016/0022-247X(83)90169-5

[36] M. M. Rahman, Multiplication and division of triangular fuzzy numbers, DIU Journal of Science and Technology 11(2) (2016), 49-53.

[37] S. Salahshour, T. Allahviranloo and S. Abbasbandy, Solving fuzzy fractional differential equations by fuzzy Laplace transforms, Communications in Nonlinear Science and Numerical Simulation 17(3) (2012), 1372-1381.

https://doi.org/10.1016/j.cnsns.2011.07.005 
[38] K. Shah and R. A. Khan, The applications of natural transform to the analytical solutions of some fractional order ordinary differential equations, Sindh University Research Journal (Science Series) 47(4) (2015), 683-686.

[39] K. Shah, A. R. Seadawy and M. Arfan, Evaluation of one dimensional fuzzy fractional partial differential equations, Alexandria Engineering Journal 59(5) (2020), 3347-3353. https://doi.org/10.1016/j.aej.2020.05.003

[40] E. Sousa and C. Li, A weighted finite difference method for the fractional diffusion equation based on the Riemann-Liouville derivative, Applied Numerical Mathematics 90(1) (2015), 22-37. https://doi.org/10.1016/j.apnum.2014.11.007

[41] R. A. Spinelli, Numerical inversion of a Laplace transform, The Journal of Chemical Physics 110(23) (1999), 636-649. https://doi.org/10.1063/1.479059

[42] C. J. Tranter, The use of the Mellin transform in finding the stress distribution in an infinite wedge, The Quarterly Journal of Mechanics and Applied Mathematics 1(1) (1948), 125-130. https://doi.org/10.1093/qjmam/1.1.125

[43] H. C. Wu, The improper fuzzy Riemann integral and its numerical integration, Information Sciences 111(1-4) (1998), 109-137. https://doi.org/10.1016/S0020-0255(98)00016-4

[44] L. A. Zadeh, Fuzzy sets, Information and Control 8(3) (1965), 338-353. https://doi.org/10.1016/S0019-9958(65)90241-X

This is an open access article distributed under the terms of the Creative Commons Attribution License (http://creativecommons.org/licenses/by/4.0/), which permits unrestricted, use, distribution and reproduction in any medium, or format for any purpose, even commercially provided the work is properly cited. 